\title{
Difference in sensitivity between SARS-CoV-2-specific $T$ cell assays in patients with underlying conditions
}

To the Editor: In the paper "Rapid measurement of SARS-CoV-2 spike T cells in whole blood from vaccinated and naturally infected individuals," Tan et al. stress the importance of measuring multiple immunological correlates in response to SARS-CoV-2 infection or COVID-19 vaccination (1). To avoid complex "traditional" T cell assays like interferon $\gamma$ (IFN- $\gamma$ ) enzyme-linked immune absorbent spot (ELISpot) or activation-induced marker (AIM) flow cytometry, Tan et al. assessed the performance of a $\mathrm{T}$ cell test specific for SARS-CoV-2 spike (S) protein based on stimulation of whole blood with overlapping peptides and subsequent detection of cytokines in plasma. In vaccinated and convalescent individuals, they found that the sensitivity of the whole-blood assay is comparable to that of traditional $\mathrm{T}$ cell assays, concluding that this is an attractive measure of SARS-CoV-2-specific T cell immunity.

Tan et al. performed their study in healthy individuals, but sensitivity and specificity of an alternative method to detect SARS-CoV-2-specific $\mathrm{T}$ cells could be different in a population with low responder rates. In a recent prospective multicenter study, we performed a comprehensive comparison of the immunogenicity of COVID-19 mRNA-1273 vaccination in kidney disease patients and found that kidney transplant (KTx) recipients had significantly lower SARS-CoV-2-specific T cell responses compared with controls (2). T cell responses were assessed by both commercial IFN- $\gamma$ release assay (IGRA, QuantiFERON, Qiagen) and in-house-validated IFN- $\gamma$ ELISpot using overlapping peptides from the $S$ protein. In a subset of participants, we found moderate correlations between the two assays in both controls and KTx recipients. The IFN- $\gamma$ ELISpot identified a T cell response in 18 out of 23 controls (78\%) and 12 out of $31 \mathrm{KTx}$ recipients (39\%). Strikingly, 16 out of $18(89 \%)$ responders were confirmed by IGRA in controls, whereas only 5 out of 12 (42\%) responders were confirmed in KTx recipients (Figure 1).
Although the IGRA is an accurate measure of specific T cell responses in healthy individuals in both our study and the study by Tan et al., our data indicate that $\mathrm{T}$ cell responses measured in whole blood of KTx recipients should be interpreted with caution. Similar results are to be anticipated in other populations with other underlying conditions or on immunosuppressive medication that lead to low $\mathrm{T}$ cell responses. Immunity assessed in whole blood by IGRA can be influenced by low lymphocyte counts (volume-based assay versus standardized cell input in ELISpot) or the presence of immunosuppressive medication in whole blood $(3,4)$. Accurate "traditional" methods to detect SARS-CoV-2-specific $\mathrm{T}$ cell responses therefore remain crucial in assessing the cellular immune response, but the IGRA is an attractive alternative for a quick screen of induction of specific $\mathrm{T}$ cell responses in large trials.

\section{Acknowledgments}

See Supplemental Acknowledgments for consortium details. This work was supported with a grant from ZonMW, The Netherlands Organization for Health Research and Development (project number 10430072010002).

\section{Rory D. de Vries, ${ }^{1}$ Marieke van der Heiden, ${ }^{2}$ Daryl Geers, ${ }^{1}$ Celine Imhof, ${ }^{3}$ Debbie van Baarle, ${ }^{2,4}$ and RECOVAC-IR Collaborators ${ }^{5}$}

${ }^{1}$ Department Viroscience, Erasmus MC, Rotterdam, Netherlands. ${ }^{2}$ Department of Medical Microbiology and Infection Prevention and ${ }^{3}$ Division of Nephrology, Department of Internal Medicine, University Medical Center Groningen, Groningen, Netherlands. ${ }^{4}$ Center for Infectious Disease Control, National Institute for Public Health and the Environment, Bilthoven, Netherlands. ${ }^{5}$ The RECOVAC-IR Collaborators are detailed in Supplemental Acknowledgments.
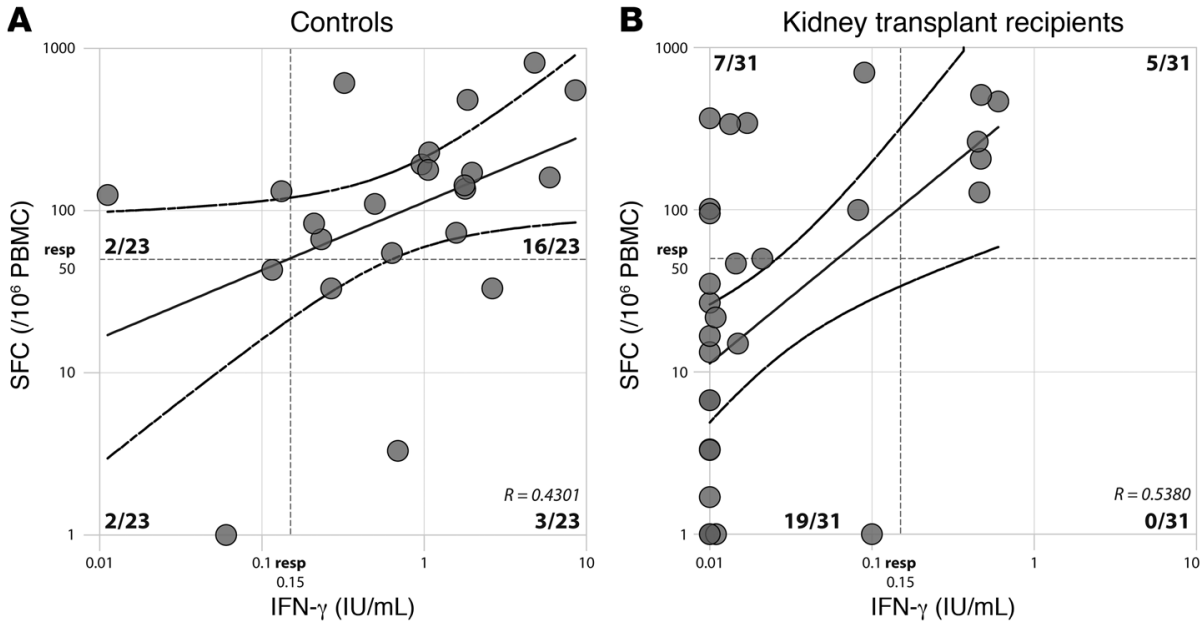

Figure 1. Correlation between IFN- $\gamma$ ELISpot and IGRA. Moderate correlation coefficients $(R)$ were observed between assays in both groups. The $x$ axis shows IGRA results (IFN- $\gamma$ ) in international units (IU)/mL in serum; the $y$ axis shows ELISpot results in spot-forming colonies (SFC) $/ 10^{6}$ PBMCs. Dotted lines indicate positive cutoffs. 
1. Tan AT, et al. Rapid measurement of SARS-CoV-2 spike T cells in whole blood from vaccinated and naturally infected individuals. JClin Invest. 2021;131(17):e152379.

2. Sanders JSF, et al. The RECOVAC immune-response study: the immunogenicity, tolerability, and safety of COVID-19 vaccination in patients with chronic kidney disease, on dialysis, or living with a kidney transplant. Transplantation. 2021. https://doi.org/10.1097/TP.0000000000003983.

3. Andersson J, et al. Effects of FK506 and cyclosporin A on cytokine production studied in vitro at a single-cell level. Immunology. 1992;75(1):136-142.

4. Staatz CE, Tett SE. Clinical pharmacokinetics and pharmacodynamics of mycophenolate in solid organ transplant recipients. Clin Pharmacokinet. 2007;46(1):13-58.
Address correspondence to: Debbie van Baarle, Department of Medical Microbiology and Infection Prevention, University Medical Center Groningen, Groningen, Netherlands. Email: d.van. baarle@umcg.nl.

Conflict of interest: The authors have declared that no conflict of interest exists.

Reference information: J Clin Invest. 2021;131(24):e155499. https://doi.org/10.1172/JCI155499.

See related response: https://doi.org/10.1172/JCI155701. 